\title{
The Large Hadron Collider
}

\author{
G. Brianti and K.M. Potter
}

\author{
CERN, Geneva, Switzerland
}

Like CERN's previous project - the Large Electron Positron (LEP) collider the Large Hadron Collider (LHC) will be a synchrotron which accelerates and stores two intense beams of particles circulating in opposite directions and collides them head-on at one or two points where particle physics detectors can study the interactions. While LEP collides electrons and positrons at energies $E$ up to $100 \mathrm{GeV}$ the LHC will collide two beams of protons at energies of up to $7.7 \mathrm{TeV}$. The LHC will be installed in the same underground tunnel which at present houses LEP alone. The factor of 77 increase in energy is made possible because the heavier protons do not radiate as much energy in the form of synchrotron radiation as do the electrons of LEP.

As the circumference of the LHC is given by the existing LEP tunnel, the maximum beam energy depends only on the magnetic field which can be reached in the high quality dipole magnets needed to guide the protons around the $27 \mathrm{~km}$ of the tunnel. These magnets which will use superconducting coils of $\mathrm{NbTi}$ cooled to $1.9 \mathrm{~K}$ are the subject of a vigorous R. \& D. programme as they need to provide a field of $10 \mathrm{~T}$, some $50 \%$ higher than that presently forseen for any other accelerator for particle physics.

\section{Design Concept}

Early studies for the LHC considered colliding bunches of protons and antiprotons circulating in opposite directions in a single ring, exactly the same technique as used in the SPS collider at CERN - the machine which was used to create the $Z$ and $\mathrm{W}$ bosons whose discovery won the Nobel Prize for C. Rubbia and S. Van der Meer in 1984. But the physics reach of a collider depends on both its energy and luminosity, the latter being proportional to the rate of interactions. The LHC is designed to study quark-quark interactions whose point-like nature means that crosssections decrease as the energy $E$ increases. To maintain equally effective physics programmes the luminosity should increase at least as $E^{2}$ and to explore rare processes such as Higgs $\rightarrow \mathrm{ZZ} \rightarrow$ four muons the LHC should be able to provide luminosities in the region of $10^{34} / \mathrm{cm}^{2} \mathrm{~s}$.

Giorgio Brianti is the Associate Director for $\mathrm{Fu}$ ture Accelerators, CERN, CH-1211 Geneva 23. Keith Potter coordinates LHC experimental areas in CERN's Accelerator Directorate.

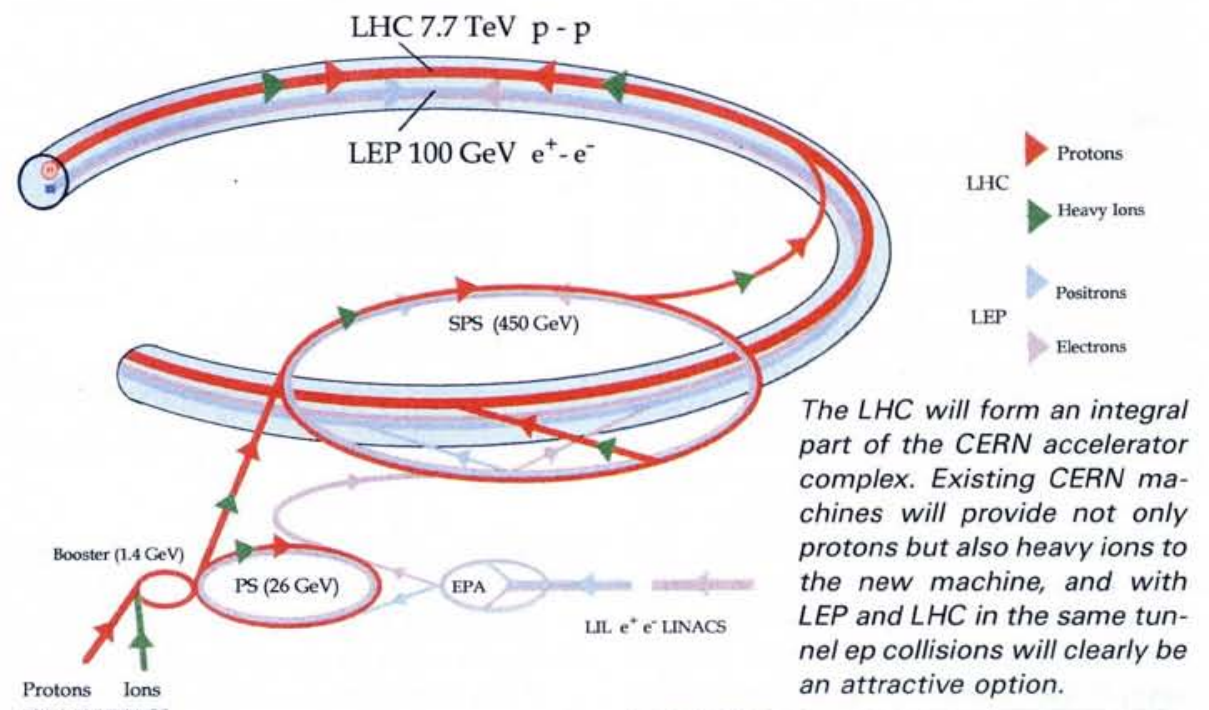

$50 \mathrm{MeV}$ LINACS

A full-size model of an LHC magnet showing how it would be installed $1.21 \mathrm{~m}$ above LEP. The sectional representation of the LHC magnet shows the two beam pipes $180 \mathrm{~mm}$ apart and slightly above the central plane of the magnet. The superconducting coils (in red) are embedded in a laminated collar (pale green) and surrounded by an iron yoke (pink). The rest of the structure, outer dewar, pipes for liquid helium and superinsulation, is related to the cryogenic system which will keep a constant flow of superfluid helium around the conductors and maintain them at $1.9 \mathrm{~K}$. (Photo CERN)

High luminosity requires high intensity circulating beams, and while antiprotons are available at CERN they cannot be produced efficiently enough to be formed into the high intensity beams required for the LHC. The only alternative is to use the principle of the CERN's decommissioned ISR machine and circulate two opposing proton beams in opposite sign magnetic channels. The LHC thus uses ingenious two-in-one magnets which provide the necessary twin channels with opposite sign fields in the same yoke and cryostat. This not only saves space, which is very important when fitting a new machine into an existing tunnel, but which also turns out to be a very economical solution with a cost saving of around $30 \%$.

The LEP collider was designed and built in a $3.76 \mathrm{~m}$ diameter tunnel in such a way as to leave space above it for a second accelerator or collider. The layout of the LHC is thus given by the form of the LEP ring which consists of eight arcs with a

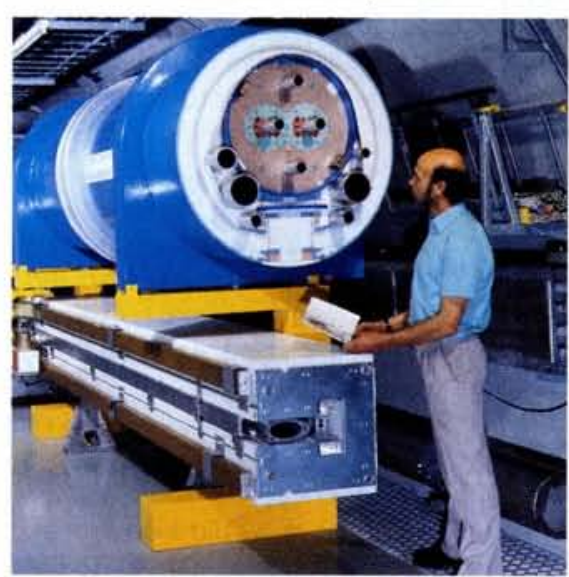

bending radius close to $3.5 \mathrm{~km}$ linked together with $550 \mathrm{~m}$ long straight sections to form a regular structure with eightfold symmetry. The two beams of the LHC will lie side by side in the horizontal plane in the arcs, about $200 \mathrm{~mm}$ apart, and will cross over in the centre of each straight section in order to not only ensure that the two rings have equal lengths but also to allow the possibility of collisions at any of the eight crossing points.

Four of these crossing points are presently occupied by the four LEP experiments but two are unused by LEP and offer excellent geological conditions for the construction of new underground caverns for LHC experiments. These two regions will be used for large general-purpose LHC detectors with the use of the existing LEP areas also possible, either when LEP experiments have been completed, or in the case of somewhat smaller LHC experiments on an alternate basis. The latter is possible because three of the 
LEP experiments can be rolled out into garage positions. The remaining two straight sections will be used for LHC machine facilities, one to provide a safe external beam dump for the beams at the end of each run, and the other a beam cleaning system which will be needed to ensure that halo particles cannot reach the vacuum chamber walls and deposit most of their energy in the superconducting magnets.

\section{Injector Facility}

The LHC will obtain its protons from the existing CERN accelerator chain of 50 $\mathrm{MeV}$ linac, $1.4 \mathrm{GeV}$ booster, $26 \mathrm{GeV}$ PS (Proton Synchrotron), and the $450 \mathrm{GeV}$ SPS (Super Proton Synchrotron). Only minor modifications and development will be needed apart from two new injection lines required to bring protons from the SPS to appropriate points on the LHC circumference The scheme ensures that no magnetic fields have to change sign during the filling cycle for both rings. The requirements of the LHC (large numbers of high intensity proton bunches with the correct emittance) are only slightly beyond the routine performance of this injector chain and the necessary development is certainly possible in the next few years. As these same injectors frequently operate with other particles, notably heavy ions such as oxygen and sulphur, the $\mathrm{LHC}$ will also be able to accelerate and collide heavy ions. There is a development programme designed to make lead ions available at CERN in the next two years, and with the expected intensities of lead ions, luminosities above $10^{25} / \mathrm{cm}^{2} \mathrm{~s}$ should be reached in the LHC with the total energy of the nuclei collisions being over $1250 \mathrm{TeV}$.

In a future phase it would also be easy to collide the LEP electron beam at $60 \mathrm{GeV}$ with the 7.7 TeV LHC proton beam, the only additional requirement being a modification to LEP at the intended collision point to raise the electron beam by the $1.21 \mathrm{~m}$ which will vertically separate the two colliders. Such an arrangement would offer up to $1.36 \mathrm{TeV}$ in the centre of mass system, four times that available at the recently commissioned ep collider HERA at the DESY Laboratory in Hamburg. The initial estimates of the luminosity which could be achieved in this way are in the $10^{32} / \mathrm{cm}^{2} \mathrm{~s}$ region.

\section{Performance}

Once the maximum collision energy is given the next most important parameter of any collider is the luminosity or collision rate it offers. Luminosity $L$ is defined by

$$
N_{x}=L \sigma_{x}
$$

where $N_{x}$ is the rate of interactions for the process with nuclear cross-section $\sigma_{x}$ and is usually quoted in units of $1 / \mathrm{cm}^{2} \mathrm{~s}$ in the case of proton colliders. In terms of the parameters of the collider, the lumino-

\section{LHC pp Performance}

$\begin{array}{lll}\text { Maximum beam energy } & E \text { for } B=9.5 \mathrm{~T} & 7.7 \mathrm{TeV} \\ \text { Centre of mass collision energy } & 2 E & 15.4 \mathrm{TeV} \\ \text { Luminosity } & L & 1.65 \times 10^{34} / \mathrm{cm}^{2} \mathrm{~s} \\ \text { Number of bunches } & k & 4725 \\ \text { Bunch spacing } & b, \Delta t & 4.5 \mathrm{~m}, 15 \mathrm{~ns} \\ \text { Number of collision points } & k_{\mathrm{x}} & 3 \\ \text { Betatron function at collision points } & \beta^{*} & 0.5 \mathrm{~m} \\ \text { Normalised emittance } & \varepsilon_{\mathrm{n}}=\sigma^{2} \gamma / \beta & 3.75 \mu \mathrm{m} \\ \text { Beam radius at collision (rms value) } & & 15 \mu \mathrm{m} \\ \text { Bunch length } & \sigma_{\mathrm{s}} & 0.075 \mathrm{~m} \\ \text { Energy spread } & \sigma_{E} / E & 1.05 \times 10^{-4} \\ \text { Horizontal crossing angle } & \phi & 200 \mu \mathrm{rad} \\ \text { Particles per bunch } & N & 1011 \\ \text { Beam-Beam tune shift parameter } & \zeta & 0.0032 \\ \text { Circulating current } & I & 0.85 \mathrm{~A} \\ \text { Stored beam energy } & U & 583 \mathrm{MJ} \\ \text { Synchrotron radiation power } & P_{\mathrm{s}} & 18.2 \mathrm{~kW}\end{array}$

sity per collision point with two equal beams is given by:

$$
L=N^{2} k f \gamma / 4 \pi \varepsilon_{n} \beta^{*}
$$

where $N$ is the number of protons in each of $k$ circulating bunches, $f$ is the frequency of revolution, $\beta^{*}$ is the value of the betatron function at the collision point and $\varepsilon_{\mathrm{n}}$ is the emittance corresponding to the $1 \sigma$ contour of the beam, normalised by multiplication by the Lorentz factor $\gamma=$ $\left(E / m_{0} c^{2}\right)$.

It is easy to see that high luminosity requires as many high density bunches as possible with the smallest possible transverse cross-section at the collision point $\left(N\right.$ and $k$ large, $\varepsilon_{n} \beta^{*}$ small). The main limit to such a simple approach comes from the so-called beam-beam effect which is the result of the extremely non-linear fields seen by the particles of one beam as they pass through the bunches of the other. Another effect is the long-range interaction between the bunches of each beam which occurs on either side of the crossing points where the beams pass through a common length of vacuum pipe, about $100 \mathrm{~m}$ long. This long-range beambeam effect is reduced as much as possible by making the beams cross with a small horizontal angle of $200 \mathrm{mrad}$ and displacing them vertically at the non-collision crossing regions, thus separating the bunches in the transverse direction by at least $6 \sigma$ everywhere except at the desired collision points. The importance of these effects is determined by the beam-beam tune-shift parameter $\zeta=N r_{p} / 4 \pi \varepsilon_{n}$ where $r_{p}$ is the classical radius of the proton. A linear tune-shift would be easy to compensate but unfortunately while a central particle suffers a shift of $\zeta$, particles with large betatron amplitudes see a much smaller effect and the result is a spread in the tuning. This spread then makes it difficult to avoid high-order resonances which are in addition excited by the nonlinear fields.

Based on experience with existing machines it is expected that it will not be pos- sible to operate the LHC with a total beam-beam tune spread $\Delta Q$ of more than 0.02 , where in addition to the above effects which occur at each collision point there is a small contribution from the nonlinearities of the machine lattice itself. This limit and the dynamic aperture, the range of amplitudes for which particle trajectories remain confined, have been extensively explored with sophisticated particle tracking progammes. Particles have been followed for more than $10^{6}$ turns by supercomputer simulation.

It turns out that with three collision regions the beam-beam limit means that $\zeta$ cannot be larger than about 0.003 . The parameters which have been chosen to maximise the luminosity while respecting this limit are given in the table above, where another practical limit, the total beam current, has also been taken into account. The current cannot be allowed to become too large as the beam will radiate synchrotron radiation which will fall on the vacuum chamber of the machine. The resulting energy deposit corresponding to a total power of $9 \mathrm{kw}$ per beam must then be removed at a temperature of a few degrees Kelvin - a highly inefficient and expensive process. In addition, the stored energy in the beam becomes very large: it can be seen in the table to be $\approx 600 \mathrm{MJ}$, already close to the limit of what can be safely absorbed by a classical beam dump.

\section{Novel Systems}

\section{Magnetic lattice}

The LHC lattice is constructed around a regular focussing-straight-defocussingstraight (FODO) cell approximately $100 \mathrm{~m}$ long. Each arc consists of 24 such cells and each half-cell consists of three $13 \mathrm{~m}$ long superconducting dipoles and a quadrupole together with a combined octupole and tuning quadrupole, steering dipoles, beam position monitors, and other multipolar correction windings.

At the end of each arc is a dispersion suppressor and matching region construc- 
ted of six $10 \mathrm{~m}$ dipoles and four quadrupoles followed by two quadrupole triplets which focus the beams down to the low betatron wavelength of $0.5 \mathrm{~m}$ needed to maximise luminosity at the collision point. The two beams are merged into the same beam pipe by a pair of special dipoles between the two triplets. Hence, all the main magnets are twin-bore except the final triplet. The general-purpose low-beta insertion can provide a beta value between 0.5 and $15 \mathrm{~m}$ and the free space for experiments is some $16 \mathrm{~m}$ on each side of the collision point.

\section{Superconducting beam magnets}

For the reasons given above the LHC requires the highest possible magnetic field which makes the superconducting dipoles the most technologically challenging components. The development of these magnets was started in 1985, in close collaboration with European industry and other accelerator laboratories, with the aim of achieving $10 \mathrm{~T}$ fields. Since then progress has been impressive (see box). The developments in superconducting cable manufacture and the latest results, which have all been obtained with magnets built in industry, show that a 9.5 T operating field, as needed to achieve the design aim of $15.4 \mathrm{TeV}$ in LHC collisions, is well within reach. In fact the manufacture of seven $10 \mathrm{~m}$ prototypes is well advanced and delivery is expected in time for testing early next year. The same magnets will then be used in a string-test to verify the cryogenic system needed to bathe the coils of all 1344 dipoles and some 2000 smaller magnets in superfluid helium and maintain them at $1.9 \mathrm{~K}$.

\section{Liquid helium cryogenics}

The massive cryogenic system needed will be based on that already being installed to cool the superconducting accelerating cavities of the LEP 200 project (the upgrading of LEP to $200 \mathrm{GeV}$ ). The system will need considerable modification, however, to lower the temperature from the $4.5 \mathrm{~K}$ used for LEP cavities to the $1.9 \mathrm{~K}$ needed for the LHC magnets and to provide the increased cooling power needed to cool down 31000 tonnes of cold mass in a reasonable time. During operation the cryogenic system has to remove not only heat from heat leaks but also that resulting from ohmic dissipation in the magnet windings, synchrotron radiation from the beam, ohmic heating in the beam-pipe, and particle losses in the magnets. A total of about 400000 litres of liquid helium will be needed but nonetheless the LHC will be extremely energy efficient providing 20 times the collision energy and 10000 times the luminosity of the SPS for a similar energy consumption.

\section{Beam cleaning}

During the whole LHC operation cycle of particle injection, acceleration, colli-

\section{LHC Superconducting Dipoles Advance}

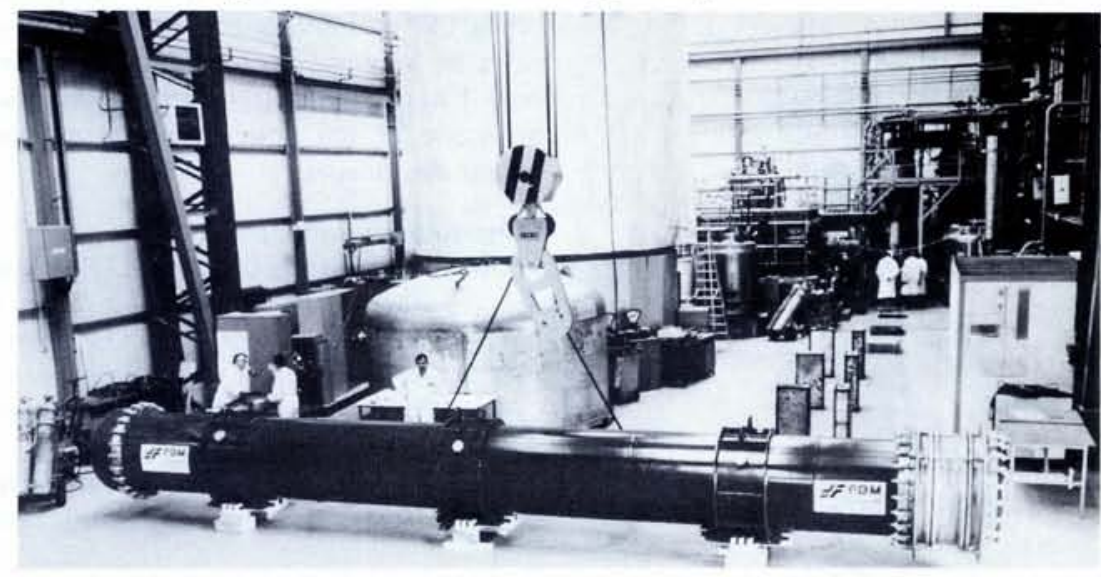

The first 10 metre twin-aperture bending magnet prior to testing at the Saclay Laboratory in France. This magnet which was built by industry with HERA type coils behaved at $4.5 \mathrm{~K}$ in exactly the same way as the best HERA single aperture magnets. When cooled to $1.9 \mathrm{~K}$ it reached its short-sample field of $8.3 \mathrm{~T}$ after only four quenches.

(Photo Saclay)

Two $1 \mathrm{~m}$ long twin-aperture dipole magnets for the LHC magnets, constructed with a cable made with $\mathrm{NbTi}$ superconductor, were tested at $1.9 \mathrm{~K}$ and a central field of $10 \mathrm{~T}$ obtained. The main effort is now to reduce the training which these first twinaperture models showed. Training is said to occur when superconducting magnets quench (a section of the conductor loses its superconducting property) prematurely, perhaps because of movements of the conductor. When the magnet is re-energised subsequent quenches occur at higher fields until eventually the field and current in the conductor reach the limiting values which make even a short sample of the cable lose its superconducting properties.

With the use of a novel technique based on large numbers of pick-up coils inside the magnet aperture it has been possible to establish that most of these training quenches occur in the ends of the coils where the mechanical support is very difficult. Several of these short models are now being rebuilt with small modifications designed to reduce the training to acceptable levels.

The field quality of the magnet design has been verified by measurements on these models and confidence in the twin-aperture design has been boosted by results obtained with a $10 \mathrm{~m}$ long magnet built with a twin bore but using large aperture coils, $75 \mathrm{~mm}$ instead of $56 \mathrm{~mm}$, of the type made for HERA magnets. This magnet behaved exactly like the standard single-bore HERA magnets at $4.2 \mathrm{~K}$ and reached a field of $8.3 \mathrm{~T}$, corresponding to the short sample limit of the superconducting cable, after only four quenches when cooled to $1.9 \mathrm{~K}$.

ding, and finally extracting and dumping the beams, one of the most critical components will be the beam cleaning system. A series of collimators in one of the straight sections must intercept the beam halo particles and prevent them depositing energy in the coils of the magnets and causing quenches. It is estimated that as few as $10^{6} \mathrm{LHC}$ protons/s could quench a magnet, while the losses from small-angle elastic scattering in the collision regions alone will scatter of the order of $10^{9}$ protons/s into the halo region.

The required halo elimination efficiency of $99.9 \%$ can only be obtained with a three-stage system of very carefully placed, $30 \mathrm{~cm}$ long absorber blocks made of highly polished tungsten. The second and third stage blocks must be positioned so as to intercept particles scattered out of the primary collimator by multiple Coulomb scattering. A particularly large proportion of the incident halo particles will be scattered in this way because of the tiny impact parameter, of the order of microns, with which they will strike the primary collimator.

\section{Beam dump}

At the end of a colliding beam run the two beams will be deflected by very fast kicker magnets, providing $18 \mathrm{Tm}$ with a $3 \mu \mathrm{s}$ rise time, into extraction channels leading to dump blocks after about $750 \mathrm{~m}$. An appropriate gap will be left in the normal bunch structure during injection so that with proper time synchronisation the kicker magnet can extract the beam without excessive losses. The extraction channels will have to be equipped with sweeping magnets to spread the beams over the dump blocks. In this way the $600 \mathrm{MJ}$ of each beam can be safely absorbed without any risk of destroying the absorber block with the thermal shock. The absorbers will be $14 \mathrm{~m}$ long blocks of iron with central cores of graphite encased in aluminium. The iron will be water cooled 


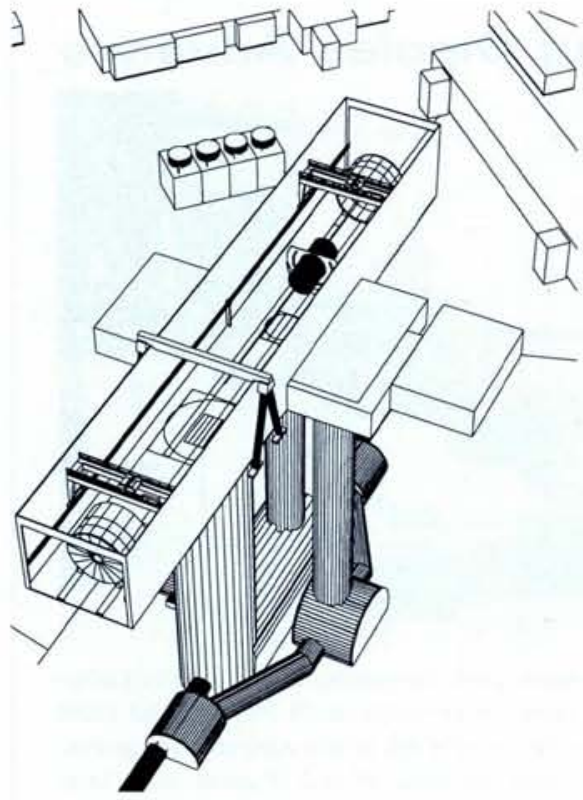

A design concept for one of the new experimental areas. The components of the experiment, a coil and return yoke of a big solenoid magnet are indicated in this drawing, will be assembled in a surface hall and then lowered down an $18 \mathrm{~m}$ diameter vertical shaft directly into the cavern on the beamline, $100 \mathrm{~m}$ underground. This cylindrical design of cavern with two access shafts could be up to $30 \mathrm{~m}$ in diameter and $70 \mathrm{~m}$ long. The collision point would be positioned close to the centre of the cavern. An alternative possibility for the design consists of a larger $30 \mathrm{~m}$ diameter vertical shaft directly over the crossing point, so that virtually no underground horizontal translation of heavy components would be needed.

to allow repeated use during setting up and machine studies and the assemblies will be installed in caverns, designed to take account of the induced radioactivity. Like most of the rest of the LHC, these caverns will be some $100 \mathrm{~m}$ underground.

\section{Experimental Caverns}

The major civil engineering work for the LHC will be the construction of new underground caverns for two, large, generalpurpose LHC experiments. The experimental programme is not yet defined but enough is known about the proposed experiments to allow the design of caverns of about $30 \mathrm{~m}$ diameter up to $70 \mathrm{~m}$ long similar to those used for LEP. The experiments will be too large and heavy to roll on rails from garage positions, as in the case of three LEP experiments. Instead they will be partially assembled on the surface and lowered in large sections, up to 3000 tons at a time. In this way installation of the experiments can be very rapid and matched to the installation time of the LHC itself. An extended shutdown of LEP of about eighteen months is expected to be suffi- cient for the final installation phase because most of the surface and underground constructions can be completed while LEP continues to operate. This is possible because the LEP beams require only $1 \mathrm{~m}$ of shielding. Good use will also be made of the normal annual maintenance shutdowns.

\section{Construction and Operation}

Once installed the LHC and LEP will be able to operate on an alternate basis if continued physics interest in LEP merits this. In any case, the possibility to operate LEP will be maintained in order to retain the ep option.

The CERN Council, which agreed in December 1991 that "the LHC is the right machine for the advance of the subject and of the future of CERN" requested a detailed programme and cost estimate for the end of 1993. It should then also be possible to offer convincing proof of the technical feasibility of the project and that the design aims with respect to magnet performance can be achieved. Technical studies suggest that with adequate funding the LHC could be built and installed in about five years while fully exploiting the physics potential of existing CERN programmes including LEP 200.

\section{FURTHER READING}

Design Study for the Large Hadron Collider, CERN Report 91-03 (1991).

Europhys. News 22 (1991) 90.

\section{UPPSALA UNIVERSITY}

Applications are invited for the position of

\section{Director at the The Svedberg Laboratory Uppsala University, Sweden}

The The Svedberg Laboratory (TSL) is a Swedish national research facility for accelerator based research operated by Uppsala University. TSL provides a range of ion beams for Swedish and international scientists with approved projects and it also carries out research using its own resources. The laboratory runs three accelerators: one combined synchrotron and storage ring (CELSIUS) with electron cooling, one $\mathrm{K}=200 \mathrm{MeV}$ cyclotron, isochronous or frequency-modulated, and one tandem van der Graaff accelerator. The highest beam energy is 1360 $\mathrm{MeV}$ for protons accelerated in CELSIUS. Also light heavy ions are available.

Ten scientists form the laboratory research staff and approximately 50 people are employed in total. About 50 research projects are at present in progress, performed by more than 200 Ph.D. scientists. Examples of active fields of research are production processes and rare decays of mesons, intermediate energy heavy-ion physics, radiative processes in intermediate energy physics, nuclear spectroscopy, neutron physics, accelerator mass spectroscopy, electronic sputtering and desorption, radiation biology, and biomedical research. The laboratory has a nationally recruited board and a Programme Advisory Committee of international experts.

The Director is appointed by the board of Uppsala University for a period of six years.

The Director, together with the Programme Advisory Committee and the board of TSL, is responsible for the scientific and technical development of the laboratory. The Director is further responsible for the day to day mangement of the laboratory.

The board of TSL has appointed a Deputy Director. The Director decides upon the division of work between him-/herself and the Deputy Director. The appointee is expected to take part in the research activities at the laboratory.

\section{Oualifications}

Documented wide experience in nuclear or particle physics research.

Established experience of managing research teams at international or large national accelerator laboratories.

\section{Other conditions}

The salary will be negotiated.

The applications together with $\mathrm{CV}$ and other documents which the applicants wish to refer to should reach Uppsala University, Box 256, S-75105 Uppsala not later than August 15th, 1992.

The Programme Advisory Committee will assist the board of TSL to forward a proposal on a new Director to the university board. 